Research Article

\title{
Electrocardiographic Characteristics of Breast Cancer Patients Treated with Chemotherapy
}

\author{
Xufei Liang, ${ }^{1}$ Yueying Wang, ${ }^{2}$ Xi Yin, ${ }^{1}$ Xiaohong Gong, ${ }^{1}$ Shuo Pan, ${ }^{1}$ Ziliang Chen, \\ and Xuhong Geng ${ }^{1}{ }^{1}$ \\ ${ }^{1}$ Department of Function, Fourth Hospital of Hebei Medical University, Shijiazhuang 050011, China \\ ${ }^{2}$ Graduate School of Medicine, Tianjin Medical University, Tianjin 300070, China \\ Correspondence should be addressed to Xuhong Geng; sjzgengxuhong@163.com
}

Received 19 October 2020; Revised 8 November 2020; Accepted 24 November 2020; Published 9 December 2020

Academic Editor: Tong Liu

Copyright ( $\odot 2020$ Xufei Liang et al. This is an open access article distributed under the Creative Commons Attribution License, which permits unrestricted use, distribution, and reproduction in any medium, provided the original work is properly cited.

\begin{abstract}
Introduction. Patients receiving chemotherapy for breast cancer may be at risk of developing cardiac dysfunction and electrophysiological abnormalities. The aim of this study is to evaluate alterations in electrocardiographic (ECG) parameters in breast cancer patients receiving chemotherapy. Materials and Methods. This was a prospective single-center cohort study conducted in the Fourth Hospital of Hebei Medical University, China. Participants with breast cancer referred for chemotherapy from May 1, 2019, to October 1, 2019, were invited to participate in the study. Standard 12-lead ECG and echocardiography were performed at baseline or before chemotherapy (prechemotherapy) (T0), after 1 cycle (T1), after 3 cycles (T2), and at the end of chemotherapy (T3). Results. A total of 64 patients with diagnosed breast cancer undergoing chemotherapy were included. Echocardiographic parameters showed no significant variation during the entire procedure (all $P>0.05$ ). The incidence of abnormal ECG increased from $43.75 \%$ at baseline to $65.63 \%$ at the end of chemotherapy, of which only the prevalence of fragmented QRS (fQRS) was significantly increased after the drug regimen $(26.56 \%$ to $53.13 \%)$. At the end of the treatment, heart rate, P-wave dispersion, corrected QT interval, T-peak to T-end, RR, SV1, RV5, Sokolow-Lyon index (SLI), and index of cardioelectrophysiological balance deteriorated markedly (all $P<0.05$ ). The area under the curve for SLI and QT dispersion (QTd) derived by ECG was 0.710 and 0.606 , respectively. The cutoff value with 2.12 of SLI by ECG had a sensitivity of $67.2 \%$ and specificity of $71.9 \%$ for differentiating patients after therapy from baselines. The cutoff value with 0.55 of QTd had a sensitivity of $60.9 \%$ and specificity of $60.9 \%$. Conclusions. The current study demonstrated that ECGs can be used to detect electrophysiological abnormalities in breast cancer patients receiving chemotherapy. ECG changes can reflect subclinical cardiac dysfunction before the echocardiographic abnormalities.
\end{abstract}

\section{Introduction}

One of the important side effects of chemotherapeutic agents used in patients with breast cancer is cardiotoxicity, which refers to cardiac dysfunction and heart failure [1]. AntiHER2 agents and chemotherapies (specifically anthracyclines, which are frequently used to treat HER2+ breast cancer) have been associated with increased risk of cardiotoxicity $[2,3]$. As treatment efficacy increases, there is an increasing number of patients who survive for extended periods and may receive chemotherapies for longer durations. Therefore, cancer patients increasingly require longterm management of chemotherapy-related morbidities. It is imperative to detect chemotherapy-induced cardiac injury in the early stage in order to, with the help of early pharmacologic intervention, prevent the occurrence of clinical heart failure. It has been reported that standard 12-lead electrocardiogram (ECG) enables the detection of different findings of cardiotoxicity such as sinus tachycardia, ST-T wave abnormalities, cardiac conduction disorders, QT prolongation, fragmented QRS, and cardiac arrhythmia during chemotherapies in cancer patients $[1,4,5]$. The 12lead ECG remains a routine screening tool owing to its noninvasive, rapid, and inexpensive properties, and it has demonstrated promise as a tool for measuring subclinical cardiotoxicity [6]. The identification of patients at risk for 
cancer therapy-induced malignant arrhythmias is of exceptional clinical importance.

Previous studies have mainly focused on global left ventricular function changes during chemotherapy. However, in fact, the administration of chemotherapeutic agents may affect the cardiac electrophysiological properties before significant mechanical impairment. Therefore, we aimed to evaluate the presence or absence of ECG abnormalities in patients newly diagnosed with breast cancer following chemotherapies.

\section{Materials and Methods}

2.1. Study Population. In total, 64 eligible female patients with early-stage breast cancer were included in this singlecenter, prospective observational clinical study between May 2019 and December 2019. 35 patients had left-sided breast cancer, and 29 patients had right-sided breast cancer. All patients received adjuvant chemotherapy after breast cancer surgery. The exclusion criteria were age under 18 years or over 80 years, other malignancies, a previous history of chemotherapy and radiation therapy (RT), pregnancy or breastfeeding, acute myocardial infarction within the previous 6 months, symptomatic heart failure (New York Heart Association Functional Classification III-IV), left ventricular ejection fraction (LVEF) $<50 \%$, structural heart disease, serious cardiac arrhythmias, chronic use of drugs known to induce cardiac damage or arrhythmia, dialysis, permanent anticoagulation, and severe psychiatric disorders life expectancy less than 6 months. The study complied with the Helsinki Declaration, and the local institutional board of ethics approved the protocol. All participants signed informed consent before enrolment. Fourth Hospital of Hebei Medical University Research Ethics Committee approved the protocol (2020011).

2.2. Echocardiography. Echocardiography was performed by a cardiologist with experience in advanced echocardiography and trained for the requirements of the study, using standard parasternal and apical views with the frame rates of 45-75 frames/s and a GE Vivid E9 ultrasound system (GE Vingmed Ultrasound, Horten, Norway) equipped with a 2.0-4.5 MHz transducer and following current recommendations for cardiac chamber quantification in adults. Echocardiography data were collected from the department of function database [7-9]. Echocardiography was performed by the same cardiologist, who was blinded to the clinical data and electrocardiographic data.

2.3. Electrocardiography. Twelve-lead ECGs were recorded before the chemotherapy for breast cancer was started at the resting and supine position (filter: $45 \mathrm{~Hz}$, alternating current filter: $50 \mathrm{~Hz}$, paper speed: $25 \mathrm{~mm} / \mathrm{s}$, and amplitude $10 \mathrm{~mm} /$ $\mathrm{mV}$; Huanan Medical, Zhengzhou, China). All of the ECGs were transferred to a personal computer to decrease error measurements and then used for $400 \%$ magnification by Adobe Photoshop software. All of the measurements were performed on the screen by manual method. No patient had fewer than nine measurable leads, and all precordial derivations were included in the measurements.

The following automated ECG measurements were extracted: heart rate (HR), P-wave amplitude (PWA), QT interval (QTI), RR interval (RR), corrected QT interval (QTc), QRS duration (QRSD), PR interval (PRI), QRS axis, and index of cardioelectrophysiological balance (iCEB: QT/ QRS [10]). The following variables were manually measured: P-wave dispersion (Pd), QT dispersion (QTd), and T-peak to T-end (TpTe). ST-T changes were analyzed according to the criteria of parameter measurement, and ECG diagnosis is based on the recommendation of the American Heart Association (AHA) (AHA/ACCF/HRS, 2007-2009) [11]. Criteria for ST-T changes were any of the following: (1) STsegment abnormalities: the ST segment was measured at $80 \mathrm{~ms}$ after J point, and the meaningful change was described as ST-segment depression $\geq 0.05 \mathrm{mV}$, or ST-segment elevation $\geq 0.10 \mathrm{mV}$ in the limb leads and/or $\geq 0.20 \mathrm{mV}$ in the chest leads. (2) T-wave changes: (a) high and sharp T-wave: the peak of $\mathrm{T}$-wave was $>0.5 \mathrm{mV}$ in the limb leads and/or $>1.5 \mathrm{mV}$ in the chest leads; (b) low and flat T-wave: the peak of T-wave was $<0.1 \mathrm{mV}$ in the limb leads or $<0.2 \mathrm{mV}$ in the chest leads; (c) bidirectional T-wave; and (d) inversed T-wave (inversion depth $\geq 0.1 \mathrm{mV}$ ). Fragmented QRS (fQRS) is defined as the presence of an additional R-wave $\left(\mathrm{R}^{\prime}\right)$, $\mathrm{R}$-wave, or the $\mathrm{S}$-wave notching, or the presence of more than one $\mathrm{R}^{\prime}$-wave in two consecutive leads [12]. An ECG is classified as abnormal if the following features were detected: sinus arrhythmia, atrial fibrillation, premature atrial or ventricular contraction, atrioventricular block, fQRS, ST segment, or T-wave changes. ECG parameters of the patients were measured by two blinded independent cardiologists (Y. W and Z. C), and ECGs were evaluated by a third independent reviewer (X. G) when there was a discrepancy between the evaluations of the two readers. For each study patient, these values were calculated on average three times.

2.4. Statistical Analysis. Continuous variables were summarized by the median and interquartile range or mean\pm standard deviation and compared by one-way analysis of variance (ANOVA) or Fisher's exact test; otherwise, median and interquartile range (IQR) were reported. Categorical variables were expressed as frequencies and percentages and compared using the chi-square tests. The area under the receiver operating characteristic (ROC) curve was calculated to determine the capability of various ECG parameters to discriminate patients after chemotherapy from baselines. IBM SPSS Statistics 22.0 (IBM Corp. Released 2013. IBM SPSS Statistics for Windows, version 22.0. Armonk, NY: IBM Corp.) was used for statistical analyses. A $P$ value of $<0.05$ was considered significant.

\section{Results}

3.1. Baseline Clinical Characteristics of Study Population. The study enrolled 64 women (mean age, $49.09 \pm 9.61$ years) with breast cancer treated with chemotherapy. The mean body mass index was $24.02 \pm 3.18 \mathrm{~kg} / \mathrm{m}^{2}$. Among 
comorbidities, diabetes mellitus was present in $4.69 \%$, hypertension in $12.5 \%$, and coronary artery disease in $3.13 \%$ of the included cases. Patients received antihypertensive drugs: angiotensin-converting enzyme inhibitor or angiotensin receptor antagonist $(1.56 \%)$ and calcium channel blockers (9.38\%). Baseline clinical characteristics of all participants are presented in Table 1 .

3.2. Echocardiography. Echocardiographic parameters are shown in Table 2 . There was no statistically significant difference $(P>0.05)$ between baseline and each follow-up point during chemotherapy.

3.3. Electrocardiography. The incidence of abnormal ECG increased from $43.75 \%$ at baseline to $65.63 \%$ at the end of the treatment (Table 3). This was mainly due to a higher proportion of patients with fQRS after chemotherapy (26.56\% to 53.13\%, $P<0.01$ ).

After three cycles of chemotherapy, heart rate (HR) (76.66 \pm 11.99 to $81.23 \pm 13.28 \mathrm{bpm}, P=0.037$ ), QRS dispersion (QTd) $(21.25 \pm 10.95$ to $27.50 \pm 13.50 \mathrm{~ms}, P<0.01)$, SV1 $(1.18 \pm 0.41$ to $1.42 \pm 0.49 \mathrm{~ms}, P<0.01)$, and Sokolow-Lyon index (SLI) $(1.92 \pm 0.59$ to $2.26 \pm 0.69 \mathrm{mV}$, $P<0.01)$ increased significantly. At the end of the treatment, HR (76.66 \pm 11.99 to $82.14 \pm 12.74 \mathrm{bpm}, P=0.013)$, P-wave dispersion (Pd) $(20.38 \pm 9.76$ to $16.81 \pm 9.41 \mathrm{~ms} P=0.029)$, corrected QT interval (QTc) (411.38 \pm 26.83 to $421.69 \pm 21.30 \mathrm{~ms}, \quad P=0.032)$, T-peak to T-end $(\mathrm{TpTe})$ $(73.63 \pm 14.20$ to $80.13 \pm 14.37 \mathrm{~ms}, \quad P=0.024), \quad$ RR $(0.80 \pm 0.12$ to $0.75 \pm 0.11 \mathrm{~s}, P=0.011)$, SV1 $(1.18 \pm 0.41$ to $1.49 \pm 0.48 \mathrm{mV}, \quad P<0.01), \quad$ RV5 $\quad(0.74 \pm 0.33 \quad$ to $0.88 \pm 0.40 \mathrm{mV}, \quad P=0.037), \quad$ SLI $\quad(1.92 \pm 0.59 \quad$ to $2.37 \pm 0.65 \mathrm{mV}, \quad P<0.01), \quad$ and $\mathrm{iCEB} \quad(4.29 \pm 0.59$ to $4.03 \pm 0.53, P=0.011$ ) deteriorated markedly (all $P<0.05$ ) (Table 4).

3.4. Receiver Operating Characteristic (ROC) Analysis. Table 5 shows the ROC curves generated using two ECG parameters to discriminate between before and after chemotherapy. Compared with the QTd, SLI had a greater area under the ROC curve and a cutoff value with 2.12 had a sensitivity of $67.2 \%$ and specificity of $71.9 \%$ for differentiating patients after chemotherapy from baselines. For QTd, the area under the ROC curve was 0.61 and a cutoff value with 0.55 had a sensitivity of $60.9 \%$ and specificity of $60.9 \%$ for differentiating patients after chemotherapy from baselines (Figure 1).

\section{Discussion}

Cardiotoxicity following chemotherapy in patients with breast cancer is a potentially life-threatening complication. Cardiac function can be assessed with echocardiography and cardiac biomarkers. However, there are few studies on electrocardiographic characteristics following chemotherapy in patients with cancer, especially breast cancer. To the best of our knowledge, no study has assessed
TABLE 1: Baseline clinical characteristics and cardiovascular risk factors in breast cancer patients.

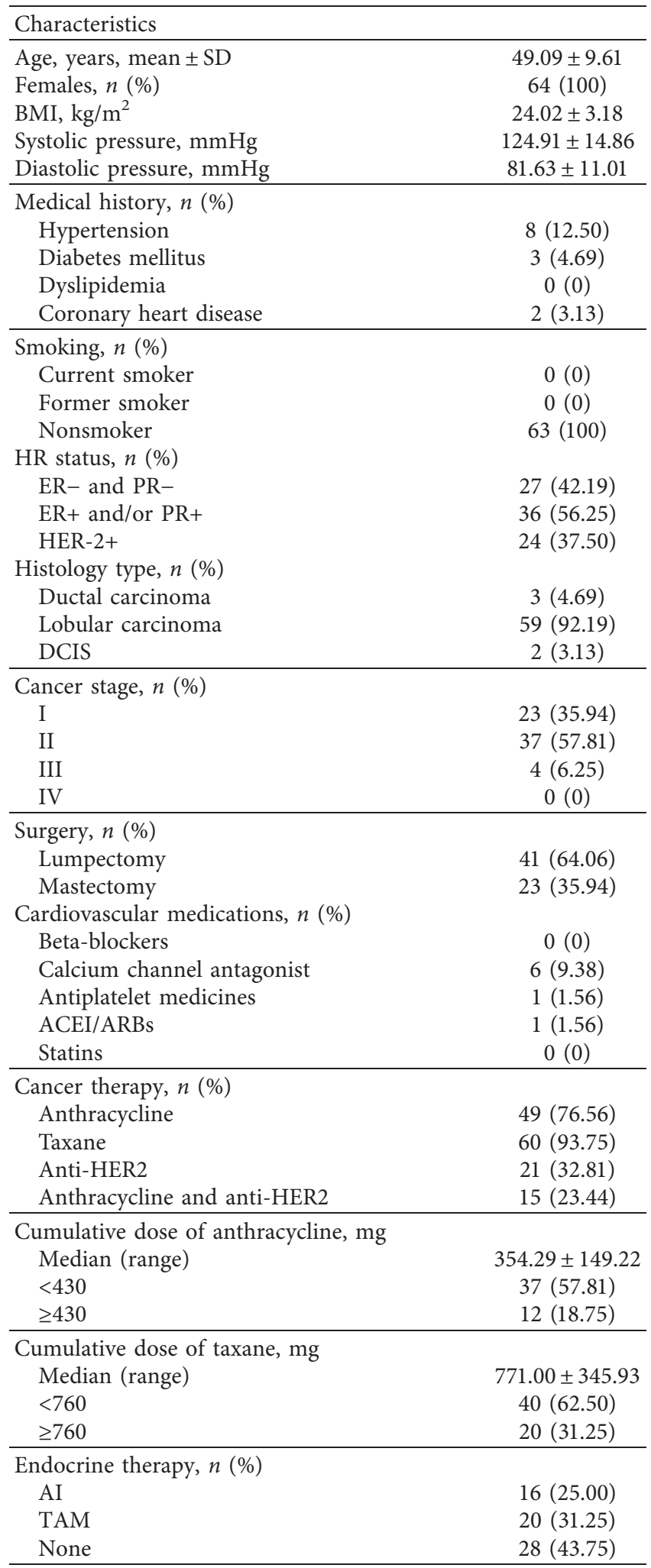

BMI, body mass index; ACEIs, angiotensin-converting enzyme inhibitors; ARBs, angiotensin receptor blockers; HER2, human epidermal growth factor receptor 2; DCIS, ductal carcinoma in situ; AI, aromatase inhibitor; BMI, body mass index; DCIS, ductal carcinoma in situ; ER, estrogen receptor; HR, hormone receptor; PR, progesterone receptor; TAM, tamoxifen. 
TABLE 2: Echocardiographic parameters before and at each follow-up point during chemotherapy in breast cancer patients.

\begin{tabular}{|c|c|c|c|c|c|c|c|}
\hline Variable & T0 & $\mathrm{T} 1$ & $P$ value & $\mathrm{T} 2$ & $P$ value & T3 & $P$ value \\
\hline LVIDd $(\mathrm{cm})$ & $4.60 \pm 0.29$ & $4.64 \pm 0.29$ & 0.422 & $4.67 \pm 0.30$ & 0.184 & $4.58 \pm 0.27$ & 0.711 \\
\hline $\mathrm{LA}(\mathrm{cm})$ & $3.00 \pm 0.31$ & $3.05 \pm 0.39$ & 0.458 & $3.06 \pm 0.27$ & 0.425 & $3.01 \pm 0.32$ & 0.919 \\
\hline LVEF (\%) & $67.00 \pm 4.07$ & $66.09 \pm 3.94$ & 0.201 & $65.95 \pm 3.96$ & 0.139 & $65.34 \pm 4.00$ & 0.052 \\
\hline E/A ratio & $1.10 \pm 0.35$ & $1.16 \pm 0.48$ & 0.389 & $1.11 \pm 0.41$ & 0.873 & $1.03 \pm 0.38$ & 0.334 \\
\hline $\mathrm{E} / \mathrm{E}^{\prime}$ ratio & $7.64 \pm 1.74$ & $7.99 \pm 1.99$ & 0.298 & $7.86 \pm 1.93$ & 0.519 & $7.28 \pm 1.96$ & 0.310 \\
\hline
\end{tabular}

Values are mean $\pm \mathrm{SD}$. " Compared with T0 $p<0.05$. T0, baseline before chemotherapy; T1, after 1 cycle of chemotherapy; T2, after 3 cycles of chemotherapy; T3, end of chemotherapy; LVIDd, left ventricular internal dimension diastole; LA, left atrial diameter; LVEF, left ventricle ejection fraction.

TABLE 3: ECG changes before and at each follow-up point during chemotherapy in BC patients.

\begin{tabular}{|c|c|c|c|c|c|c|c|}
\hline ECG changes & T0 & $\mathrm{T} 1$ & $P$ value & $\mathrm{T} 2$ & $P$ value & T3 & $P$ value \\
\hline Abnormal ECG, $n(\%)$ & $28(43.75)$ & $36(56.25)$ & 0.157 & $41(64.06)$ & $0.021^{*}$ & $42(65.63)$ & $0.013^{*}$ \\
\hline ST-T changes, $n(\%)$ & $7(10.94)$ & $10(15.63)$ & 0.435 & $10(15.62)$ & 0.435 & $10(15.62)$ & 0.435 \\
\hline ST changes & $6(9.38)$ & $7(10.94)$ & 0.770 & $7(10.94)$ & 0.770 & $7(10.94)$ & 0.770 \\
\hline T-wave changes & $1(1.56)$ & $6(9.38)$ & 0.052 & $8(12.50)$ & $0.016^{*}$ & $6(9.38)$ & 0.052 \\
\hline Arrhythmias, $n(\%)$ & $13(20.31)$ & $11(17.19)$ & 0.651 & $13(20.31)$ & 1.000 & $14(21.88)$ & 0.828 \\
\hline Sinus tachyarrhythmia & $1(1.56)$ & $3(4.69)$ & 0.310 & $5(7.81)$ & 0.094 & $6(9.38)$ & 0.052 \\
\hline Ventricular premature beats & $2(3.13)$ & $2(3.13)$ & 1.000 & $2(3.13)$ & 1.000 & $3(4.69)$ & 0.310 \\
\hline First-degree AVB & $3(4.69)$ & $2(3.13)$ & 0.648 & $1(1.56)$ & 0.310 & $1(1.56)$ & 0.310 \\
\hline Intraventricular block & $1(1.56)$ & $1(1.56)$ & 1.000 & $1(1.56)$ & 1.000 & $1(1.56)$ & 1.000 \\
\hline QTc prolongation, $n(\%)$ & $3(4.69)$ & $4(6.25)$ & 0.697 & $1(1.56)$ & 0.310 & $2(3.13)$ & 0.648 \\
\hline fQRS, $n(\%)$ & $17(26.56)$ & $27(42.19)$ & 0.063 & $30(46.88)$ & $0.017^{*}$ & $34(53.13)$ & $<0.01^{*}$ \\
\hline
\end{tabular}

Values are mean \pm SD. ${ }^{*}$ Compared with T0, $p<0.05$. T0, baseline before chemotherapy; T1, after 1 cycle of chemotherapy; T2, after 3 cycles of chemotherapy; T3, end of chemotherapy; AVB, atrioventricular block; QTc, corrected QT interval; fQRS, fragmented QRS.

TABLE 4: Electrocardiographic parameters before and at each follow-up point during chemotherapy in BC patients.

\begin{tabular}{|c|c|c|c|c|c|c|c|}
\hline Variables & T0 & $\mathrm{T} 1$ & $P$ value & $\mathrm{T} 2$ & $P$ value & T3 & $P$ value \\
\hline HR (bpm) & $76.66 \pm 11.99$ & $78.83 \pm 11.30$ & 0.321 & $81.23 \pm 13.28$ & $0.037^{*}$ & $82.14 \pm 12.74$ & 0.013* \\
\hline PWA (mV) & $0.11 \pm 0.03$ & $0.12 \pm 0.03$ & 0.454 & $0.12 \pm 0.04$ & 0.623 & $0.12 \pm 0.04$ & 0.438 \\
\hline PWD (ms) & $95.53 \pm 12.05$ & $95.61 \pm 11.60$ & 0.973 & $94.06 \pm 11.87$ & 0.529 & $96.19 \pm 16.58$ & 0.779 \\
\hline PRI (ms) & $148.97 \pm 20.37$ & $148.05 \pm 21.29$ & 0.835 & $147.78 \pm 21.54$ & 0.788 & $149.58 \pm 34.16$ & 0.890 \\
\hline $\mathrm{Pd}(\mathrm{ms})$ & $20.38 \pm 9.76$ & $18.38 \pm 9.66$ & 0.218 & $18.31 \pm 8.75$ & 0.204 & $16.81 \pm 9.41$ & $0.029^{*}$ \\
\hline QRS axis $\left({ }^{\circ}\right)$ & $39.33 \pm 30.13$ & $37.83 \pm 30.28$ & 0.769 & $40.29 \pm 24.74$ & 0.853 & $37.49 \pm 29.51$ & 0.720 \\
\hline QRSD (ms) & $87.05 \pm 13.88$ & $87.02 \pm 12.88$ & 0.989 & $89.14 \pm 12.66$ & 0.371 & $91.64 \pm 13.43$ & 0.050 \\
\hline QTc (ms) & $411.38 \pm 26.83$ & $415.61 \pm 26.67$ & 0.378 & $414.94 \pm 32.45$ & 0.458 & $421.69 \pm 21.30$ & $0.032^{*}$ \\
\hline QTd (ms) & $21.25 \pm 10.95$ & $24.75 \pm 11.92$ & 0.102 & $27.50 \pm 13.50$ & $<0.01^{*}$ & $24.94 \pm 11.71$ & 0.085 \\
\hline $\mathrm{TpTe}(\mathrm{ms})$ & $73.63 \pm 14.20$ & $76.31 \pm 18.64$ & 0.386 & $76.69 \pm 21.64$ & 0.323 & $80.13 \pm 14.37$ & $0.037^{*}$ \\
\hline $\mathrm{RR}(\mathrm{s})$ & $0.80 \pm 0.12$ & $0.78 \pm 0.11$ & 0.252 & $0.76 \pm 0.12$ & 0.042 & $0.75 \pm 0.11$ & $0.011^{*}$ \\
\hline $\mathrm{SV} 1(\mathrm{mV})$ & $1.18 \pm 0.41$ & $1.30 \pm 0.41$ & 0.144 & $1.42 \pm 0.49$ & $<0.01^{*}$ & $1.49 \pm 0.48$ & $<0.01^{*}$ \\
\hline RV5 (mV) & $0.74 \pm 0.33$ & $0.79 \pm 0.38$ & 0.512 & $0.84 \pm 0.42$ & 0.158 & $0.88 \pm 0.40$ & $0.037^{*}$ \\
\hline SLI $(\mathrm{mV})$ & $1.92 \pm 0.59$ & $2.08 \pm 0.61$ & 0.151 & $2.26 \pm 0.69$ & $<0.01^{*}$ & $2.37 \pm 0.65$ & $<0.01^{*}$ \\
\hline iCEB & $4.29 \pm 0.59$ & $4.26 \pm 0.54$ & 0.781 & $4.10 \pm 0.62$ & 0.069 & $4.03 \pm 0.53$ & $0.011^{*}$ \\
\hline
\end{tabular}

Values are mean $\pm \mathrm{SD}$. ${ }^{*}$ Compared with $\mathrm{T} 0, p<0.05$. T0, baseline before chemotherapy; T1, after 1 cycle of chemotherapy; T2, after 3 cycles of chemotherapy; T3, end of chemotherapy; HR, heart rate; PWA, P-wave amplitude; PWD, P-wave duration; PRI, PR interval; Pd, P-wave dispersion; QRSD, QRS duration; QTc, corrected QT interval; QTd, QRS dispersion; TpTe, T-peak to T-end; SLI, Sokolow-Lyon index; iCEB: index of cardioelectrophysiological balance.

electrocardiographic parameters immediately after completion of chemotherapy infusion.

Our study used ECGs to evaluate the cardiac electrophysiological changes in patients with breast cancer who received chemotherapy. The main findings of this study are as follows: (1) the incidence of abnormal ECG increased from $43.8 \%$ at baseline to $65.6 \%$ during follow-up, and this was mainly due to a higher proportion of patients with fQRS; (2) HR, Pd, QTc, TpTe, RR, SV1, RV5, SLI, and iCEB deteriorated markedly along with chemotherapy; and (3) QTd and SLI had high sensitivity and specificity in differentiating
TABLE 5: ROC curve analyses of electrocardiographic parameters.

\begin{tabular}{lccccc}
\hline Variable & AUC & 95\% CI & $\begin{array}{c}\text { Cutoff } \\
\text { value }\end{array}$ & Sensitivity & Specificity \\
\hline SLI & 0.710 & $0.620-0.799$ & 2.12 & 0.672 & 0.719 \\
QTd & 0.606 & $0.507-0.704$ & 0.55 & 0.609 & 0.609 \\
\hline
\end{tabular}

ROC, receiver operating characteristic; AUC, area under the curve; CI, confidence interval; SLI, Sokolow-Lyon index; QTd, QRS dispersion.

patients after therapy from baselines. These findings indicate the development of both depolarization and repolarization abnormalities following chemotherapy. 


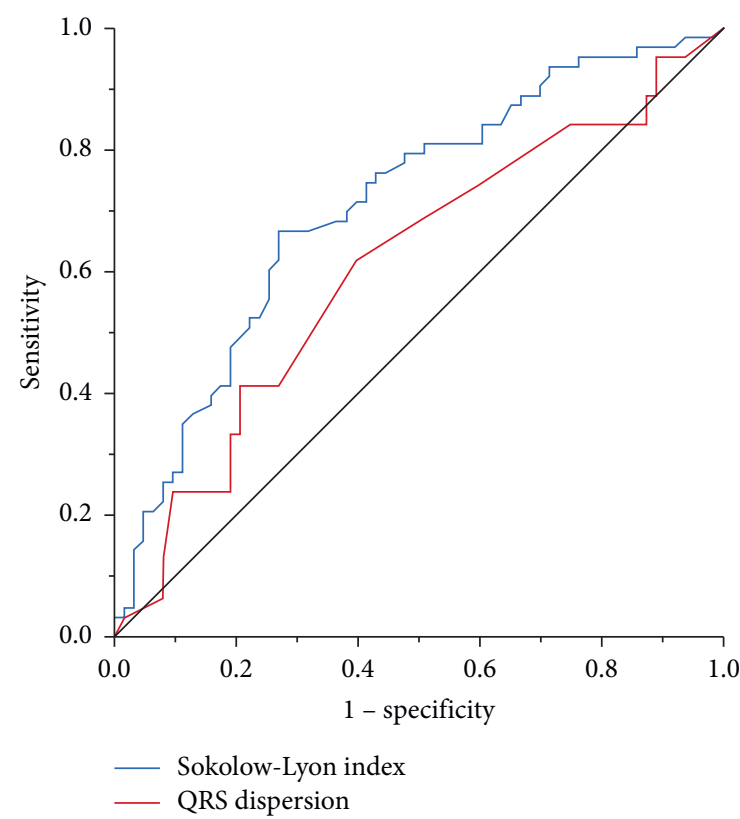

FIGURE 1: ROC curve for two electrocardiographic parameters to discriminate between pre- and posttherapy.

fQRS is a surrogate marker of myocardial conduction delay or heterogeneity with a prevalence ranging from $1 \%$ to $30 \%$ of the general population [13-15]. 26.6\% (67/252) of breast cancer patients had fQRS after anthracycline-based chemotherapy [16]. At 1-year follow-up, 19 of 52 (37.4\%) breast cancer patients receiving locoregional radiotherapy had developed fQRS on ECG [17]. Moreover, the prevalence of fQRS significantly increased in large B-cell lymphoma patients treated with anthracycline-based chemotherapy (15.8\% to $28.9 \%, P=0.041)$ [18]. We found fQRS in $53.1 \%$ of breast cancer patients after chemotherapy. In patients with coronary artery disease, fQRS has been shown to be associated with all-cause mortality and cardiac events [19]. Myocardial fibrosis may disrupt QRS morphology and lead to fragmentation of QRS on 12-lead ECG. Chemotherapeutic agents can trigger apoptosis or cause necrotic myocyte death. fQRS occurs when ventricular depolarization (VD) becomes abnormal and has been identified as an ECG biomarker of myocardial fibrosis and can be used to predict adverse cardiovascular events $[12,20]$.

In the ECG, ventricular repolarization (VR) is represented by QTc intervals, and QTc prolongations relate to a higher risk of ventricular arrhythmias in different conditions. As a risk factor for torsades de pointes (TdP) and sudden cardiac death, QTc prolongation is a toxicity of significant concern $[21,22]$. Puppe et al. found a significant increase in QTc intervals after breast cancer treatment with 4 cycles of EC-Doc regimen (epirubicin, cyclophosphamide, and docetaxel) [23]. Several investigations already demonstrated significant QTc prolongation induced by anticancer therapies, especially in anthracycline regimens [24-26]. Similar to our study, in patients with breast neoplasms undergoing chemotherapy regimen with anthracycline $(\mathrm{A}$; doxorubicin), cyclophosphamide $(\mathrm{C})$, and taxane (T; paclitaxel), Veronese et al. also observed prolongation of the
QTc interval [27]. In addition, in our study, 25.0\% and 31.3\% of breast patients were treated with aromatase inhibitor (AI) and tamoxifen (TAM), respectively. Several studies reveal the potential for endocrine therapy to induce ventricular arrhythmias, particularly TdP [28-30].

In this study, our results revealed a significantly increased QTd after chemotherapy. The QTd is defined as the difference between maximal and minimal QT intervals on a 12-lead surface ECG and reflects the regional heterogeneity of VR. Prolonged QT dispersion was even shown to predict acute heart failure in patients after high-dose cyclophosphamide therapy [31]. Further, it has been regarded as an index of ventricular arrhythmia, which may lead to sudden cardiac death [32]. Patients with breast cancer treated with trastuzumab after an anthracycline-based regimen exhibited a significantly higher QTd than nontreated patients $(0.064 \pm 0.023 \mathrm{~s}$ vs. $0.051 \pm 0.016 \mathrm{~s}$, respectively, $P=0.034)$ [33].

SLI is recommended as diagnostic screening method for left ventricular hypertrophy. In this study, we found SLI increased through chemotherapy and thus appears to represent a transitional state from a normal healthy heart to heart failure with preserved ejection fraction. iCEB can provide information about both the depolarization and repolarization phases of the cardiac action potential and is a surrogate marker of excitation wavelength. In experimental studies, a $10 \%$ variation (either increase or decrease) of $\mathrm{iCEB}$ values from baseline showed to be a promising marker for drug-induced arrhythmic risk [32, 34]. However, data from clinical trials are scarce. To date, there is no comprehensive, easy to measure, and widely available risk marker available. High iCEB values are associated with TdP and low values with non-TdP-mediated VT/VF [10]. In this study, our results revealed a decreased iCEB after chemotherapy in breast cancer. In this study, chemotherapy did not induce a significant change in LVEF. Importantly, LVEF measurement shows a low sensitivity for the early detection of subclinical cardiotoxicity [35]. This might explain why in our observation period we could not detect any decrease in LVEF despite significant electrocardiographic abnormities. Therefore, echocardiography might be suboptimal for detecting acute cardiac complications. These data supported the idea that ECG could identify mild cardiotoxicity in an earlier stage than echocardiography Whilst strain imaging can also be used for early detection of myocardial damage, the advantage of electrocardiography is their rapid and wide availability for routine clinical use. Regular ECG monitoring after initiation of chemotherapy hence is great of importance and may help cardiologists and oncologists tailor treatments during clinical works.

Several limitations of the present study should be noted. Firstly, our study investigated a small number and short follow-up of patients in a single center. Further studies are needed to verify our findings. Secondly, baseline thyroid function and history of heart failure were not collected, which may influence the ECG changes of the patients. Thirdly, the cardiac biomarkers, such as brain natriuretic peptide and troponin, were not tested in most of the included patients because it is limited by medical insurance. 
Finally, patients in this study are treated with multiple chemotherapeutics such as anthracyclines and cyclophosphamide, which may cause cardiotoxicity that is indistinguishable. Larger prospective studies examining the roles of ECG parameters for risk stratification purposes are needed in the future.

\section{Conclusions}

In this prospective study of patients with breast cancer who underwent chemotherapy, cardiotoxicity can also manifest as the emergence of ECG abnormalities, specifically abnormal ventricular repolarization. With further study, SLI and QTd ratio could potentially be used for differentiating patients after therapy from baselines. The data from this study demonstrated that ECG can be conducted to evaluate the subclinical cardiac damage for breast cancer patients after chemotherapy. ECG could help to detect subclinical cardiac dysfunction earlier than echocardiography. Regular ECG monitoring may help to detect early cardiotoxicity during follow-up following chemotherapy.

\section{Data Availability}

The data used to support the findings of this study are available from the corresponding author upon request.

\section{Conflicts of Interest}

The authors declare that there are no conflicts of interest

\section{Authors' Contributions}

Xufei Liang and Yueying Wang contributed equally. Xufei Liang and Yueying Wang investigated the study, wrote the original draft, and performed formal analysis. Xi Yin, Xiaohong Gong, Shuo Pan, and Ziliang Chen investigated the study. Xuhong Geng conceptualized the study, contributed to methodology, reviewed and edited the manuscript, and supervised the study.

\section{Acknowledgments}

This study was supported by grants ZD20140293 and ZD20140348 from Key Project Plan of Medical Science Research from Hebei Provincial Department of Health.

\section{References}

[1] J. L. Zamorano, P. Lancellotti, D. Rodriguez Muñoz et al., "2016 ESC position paper on cancer treatments and cardiovascular toxicity developed under the auspices of the ESC committee for practice guidelines," European Heart Journal, vol. 37, no. 36, pp. 2768-2801, 2016.

[2] M. U. Yood, K. E. Wells, S. H. Alford et al., "Cardiovascular outcomes in women with advanced breast cancer exposed to chemotherapy," Pharmacoepidemiology and Drug Safety, vol. 21, no. 8, pp. 818-827, 2012.

[3] H. D. Long, Y. E. Lin, J. J. Zhang, W. Z. Zhong, and R. N. Zheng, "Risk of congestive heart failure in early breast cancer patients undergoing adjuvant treatment with trastuzumab: a meta-analysis," The Oncologist, vol. 21, no. 5, pp. 547-554, 2016.

[4] T. M. Suter and M. S. Ewer, "Cancer drugs and the heart: importance and management," European Heart Journal, vol. 34, no. 15, pp. 1102-1111, 2013.

[5] M. Gurdogan and U. Ozkan, "A novel predictor of chemotherapeutic cardiotoxicity in patients with non-hodgkin lymphoma," Oncology Research and Treatment, vol. 42, no. $7 /$ 8, pp. 375-381, 2019.

[6] Q. Chen, A. Gasperetti, D. G. Della Rocca et al., "The value of baseline and arrhythmic ECG in the interpretation of arrhythmic mechanisms," Cardiac Electrophysiology Clinics, vol. 11, no. 2, pp. 219-238, 2019.

[7] R. M. Lang, M. Bierig, R. B. Devereux et al., "Recommendations for chamber quantification: a report from the American society of echocardiography's guidelines and standards committee and the chamber quantification writing group, developed in conjunction with the European association of echocardiography, a branch of the European society of cardiology," Journal of the American Society of Echocardiography, vol. 18, no. 12, pp. 1440-1463, 2005.

[8] R. M. Lang, L. P. Badano, V. Mor-Avi et al., "Recommendations for cardiac chamber quantification by echocardiography in adults: an update from the American Society of Echocardiography and the European Association of Cardiovascular Imaging," Journal of the American Society of Echocardiography, vol. 28, no. 1, pp. 1-39, 2015.

[9] M. Galderisi, B. Cosyns, T. Edvardsen et al., "Standardization of adult transthoracic echocardiography reporting in agreement with recent chamber quantification, diastolic function, and heart valve disease recommendations: an expert consensus document of the European association of cardiovascular imaging," European Heart Journal-Cardiovascular Imaging, vol. 18, no. 12, pp. 1301-1310, 2017.

[10] T. Robyns, H. R. Lu, D. J. Gallacher et al., "Evaluation of index of cardio-electrophysiological balance (iCEB) as a new biomarker for the identification of patients at increased arrhythmic risk," Annals of Noninvasive Electrocardiology, vol. 21, no. 3, pp. 294-304, 2016.

[11] P. M. Rautaharju, B. Surawicz, L. S. Gettes et al., "AHA/ ACCF/HRS recommendations for the standardization and interpretation of the electrocardiogram: part IV: the ST segment, $\mathrm{T}$ and $\mathrm{U}$ waves, and the QT interval: a scientific statement from the American heart association electrocardiography and arrhythmias committee, council on clinical cardiology; the American college of cardiology foundation; and the heart rhythm society. Endorsed by the international society for computerized electrocardiology," Journal of the American College of Cardiology, vol. 53, pp. 982-991, 2009.

[12] M. K. Das, H. Suradi, W. Maskoun et al., "Fragmented wide QRS on a 12-lead ECG," Circulation: Arrhythmia and Electrophysiology, vol. 1, no. 4, pp. 258-268, 2008.

[13] K. Narayanan, L. Zhang, C. Kim et al., "QRS fragmentation and sudden cardiac death in the obese and overweight," Journal of the American Heart Association, vol. 4, no. 3, Article ID e1654, 2015.

[14] M. K. Das, B. Khan, S. Jacob, A. Kumar, and J. Mahenthiran, "Significance of a fragmented QRS complex versus a Q wave in patients with coronary artery disease," Circulation, vol. 113, no. 21, pp. 2495-2501, 2006.

[15] N. C. Flowers, L. G. Horan, J. R. Thomas, and W. J. Tolleson, "The anatomic basis for high-frequency components in the electrocardiogram," Circulation, vol. 39, no. 4, pp. 531-539, 1969. 
[16] M. Dural, L. Demir, E. Babayiğit et al., "Fragmented QRS formation and its predictors in patients with breast cancer receiving anthracycline-based chemotherapy," Journal of Electrocardiology, vol. 54, pp. 5-9, 2019.

[17] A. Adar, E. Canyılmaz, A. Kiris et al., "Radiotherapy induces development of fragmented QRS in patients with breast cancer," Breast Care, vol. 10, no. 4, pp. 277-280, 2015.

[18] Z. Chen, K. Lu, L. Zhou et al., "Electrocardiographic characteristics of diffuse large B-cell lymphoma patients treated with anthracycline-based chemotherapy," Journal of Electrocardiology, vol. 60, pp. 195-199, 2020.

[19] M. K. Das, C. Saha, H. El Masry et al., "Fragmented QRS on a 12-lead ECG: a predictor of mortality and cardiac events in patients with coronary artery disease," Heart rhythm, vol. 4, no. 11, pp. 1385-1392, 2007.

[20] Z. Dohy, A. Vereckei, V. Horvath et al., "How are ECG parameters related to cardiac magnetic resonance images? Electrocardiographic predictors of left ventricular hypertrophy and myocardial fibrosis in hypertrophic cardiomyopathy," Annals of Noninvasive Electrocardiology, vol. 25, Article ID e12763, 2020.

[21] A. Porta-Sánchez, C. Gilbert, D. Spears et al., "Incidence, diagnosis, and management of QT prolongation induced by cancer therapies: a systematic review," Journal of the American Heart Association, vol. 6, no. 12, Article ID e007724, 2017.

[22] M. A. Armanious, S. Mishra, and M. G. Fradley, "Electrophysiologic toxicity of chemoradiation," Current Oncology Reports, vol. 20, no. 6, p. 45, 2018.

[23] J. Puppe, D. van Ooyen, J. Neise et al., "Evaluation of QTc interval prolongation in breast cancer patients after treatment with epirubicin, cyclophosphamide, and docetaxel and the influence of interobserver variation," Breast Care, vol. 12, no. 1, pp. 40-44, 2017.

[24] T. Nousiainen, E. Vanninen, A. Rantala, E. Jantunen, and J. Hartikainen, "QT dispersion and late potentials during doxorubicin therapy for non-Hodgkin's lymphoma," Journal of Internal Medicine, vol. 245, no. 4, pp. 359-364, 1999.

[25] F. Galetta, F. Franzoni, G. Cervetti et al., "Effect of epirubicinbased chemotherapy and dexrazoxane supplementation on QT dispersion in non-hodgkin lymphoma patients," Biomedicine \& Pharmacotherapy, vol. 59, no. 10, pp. 541-544, 2005.

[26] J. Duan, J. Tao, M. Zhai et al., “Anticancer drugs-related QTc prolongation, torsade de pointes and sudden death: current evidence and future research perspectives," Oncotarget, vol. 9, no. 39, pp. 25738-25749, 2018.

[27] P. Veronese, D. T. Hachul, M. I. Scanavacca et al., "Effects of anthracycline, cyclophosphamide and taxane chemotherapy on QTc measurements in patients with breast cancer," PLoS One, vol. 13, Article ID e196763, 2018.

[28] V. Grouthier, B. Lebrun-Vignes, A. M. Glazer et al., "Increased long QT and torsade de pointes reporting on tamoxifen compared with aromatase inhibitors," Heart, vol. 104, no. 22, pp. 1859-1863, 2018.

[29] D. L. Trump, D. C. Smith, P. G. Ellis et al., "High-dose oral tamoxifen, a potential multidrug-resistance-reversal agent: phase I trial in combination with vinblastine," JNCI Journal of the National Cancer Institute, vol. 84, no. 23, pp. 1811-1816, 1992.

[30] I. F. Pollack, R. C. DaRosso, P. L Robertson et al., "A phase I study of high-dose tamoxifen for the treatment of refractory malignant gliomas of childhood," Clinical Cancer Research: An Official Journal of the American Association for Cancer Research, vol. 3, no. 7, pp. 1109-1115, 1997.
[31] H. Nakamae, K. Tsumura, M. Hino, T. Hayashi, and N. Tatsumi, "QT dispersion as a predictor of acute heart failure after high-dose cyclophosphamide," The Lancet, vol. 355, no. 9206, pp. 805-806, 2000.

[32] G. Tse and B. P. Yan, "Traditional and novel electrocardiographic conduction and repolarization markers of sudden cardiac death," Europace, vol. 19, no. 5, pp. 712-721, 2017.

[33] O. Tanriverdi, N. Meydan, and S. Barutca, "Long-term effect of trastuzumab on QT dispersion in adjuvant treatment for patients with her2 receptor positive breast cancer: a pilot study," Medical Oncology, vol. 29, no. 5, pp. 3265-3271, 2012.

[34] M. Barber, L. S. Nguyen, J. Wassermann, J.-P. Spano, C. Funck-Brentano, and J.-E. Salem, "Cardiac arrhythmia considerations of hormone cancer therapies," Cardiovascular Research, vol. 115, no. 5, pp. 878-894, 2019.

[35] S. M. Swain, F. S. Whaley, and M. S. Ewer, "Congestive heart failure in patients treated with doxorubicin," Cancer, vol. 97, no. 11, pp. 2869-2879, 2003. 